\title{
Collective Intelligence for Translational Medicine: Crowdsourcing insights and innovation from an interdisciplinary biomedical research community
}

\author{
Budge E J, Tsoti S M, Howgate D J, Sivakumar S, Jalali M*
}

Eleanor Jane Budge ${ }^{1}$, Sandra Maria Tsoti ${ }^{2}$, Daniel James Howgate ${ }^{3}$, Shivan Sivakumar ${ }^{4,5}$, Morteza Jalali $6,7,8^{*}$

${ }^{1}$ Keble College, University of Oxford, Oxford, UK

${ }^{2}$ Faculty of Medicine, Imperial College London, London, UK.

${ }^{3}$ Trauma Unit, John Radcliffe Hospital, Oxford, UK.

${ }^{4}$ Department of Oncology, University of Oxford, Oxford, UK.

${ }^{5}$ Oxford Cancer and Haematology Centre, Churchill Hospital, Oxford, UK.

${ }^{6}$ Anne McLaren Laboratory for Regenerative Medicine, Wellcome Trust-Medical Research Council Cambridge Stem Cell Institute, University of Cambridge, Cambridge, UK.

${ }^{7}$ Department of Plastic and Reconstructive Surgery, Nuffield Orthopaedic Centre, Oxford, UK.

${ }^{8}$ Nuffield Department of Orthopaedics, Rheumatology and Musculoskeletal Sciences, Medical Sciences Division, University of Oxford, Oxford, UK.

${ }^{*}$ Corresponding author.

Dr Morteza Jalali

Department of Plastic and Reconstructive Surgery

Nuffield Orthopaedic Centre

Nuffield Department of Orthopaedics, Rheumatology and Musculoskeletal Sciences

Medical Sciences Division, University of Oxford

Windmill Road

Oxford OX3 7HE UK

email morteza.jalali@ndorms.ox.ac.uk 


\title{
Collective Intelligence for Translational Medicine: Crowdsourcing insights and innovation from an interdisciplinary biomedical research community
}

\begin{abstract}
Translational medicine bridges the gap between discoveries in biomedical science and their safe and effective clinical application. Despite the gross opportunity afforded by modern technology for unparalleled advances in this field, the process of translation remains protracted. Efforts to expedite science translation have included the facilitation of interdisciplinary collaboration within both academic and clinical environments in order to generate integrated working platforms fueling the sharing of knowledge, expertise and tools to align biomedical research with clinical need. However, barriers to scientific translation remain and further progress is urgently required. Collective intelligence and crowdsourcing applications offer the potential for global online networks, allowing connection and collaboration between a wide variety of fields. This would drive the alignment of biomedical science with biotechnology, clinical need and patient experience to deliver evidence-based innovation to revolutionize medical care worldwide. Here we discuss the critical steps towards implementing collective intelligence in translational medicine using the experience of those in other fields of science and public health.
\end{abstract}

\section{Key words}

Translational Medicine, Collective intelligence, Crowdsourcing, Biomedical research, Interdisciplinary collaboration, Research networks. 


\section{Key messages}

- The scientific translation of biomedical research into clinical applications is protracted, despite the mass opportunity afforded by modern science.

- Barriers to translational medicine exist as a result of the impracticalities of research, organizational hurdles and lack of an interdisciplinary workforce.

- Collective intelligence and crowdsourcing offer the potential to expedite the translational process by providing a platform upon which interdisciplinary workforces can collaborate and communicate, aligning biomedical research with clinical need to revolutionize healthcare worldwide.

\section{Introduction}

Translational medicine describes the journey of scientific discovery from the laboratory bench to the patient bedside and can be used to drive the evolution of medical practice and transform healthcare worldwide (1). Translational medicine aims to implement evidence-based research toward clinical applications such as novel diagnostic techniques, therapeutics, medical devices and implantable materials for tissue replacement and also plays a role in the development of public healthcare policies. Scientific advances created through research and development have increased our understanding of disease pathology, which in turn presents unique opportunities to develop and innovate on a greater level than ever before. Paradoxically, the translational process remains frustratingly protracted and the implementation of basic scientific research findings into clinical practice is too often not achieved within a useful and clinically relevant timeframe. Here we discuss the potential impact of the application of collective intelligence and crowdsourcing in 
translational medicine, and the experience of those doing so in fields outside of medicine.

In order to effectively utilize the knowledge gained through cutting-edge scientific research, it is essential to understand the current barriers to translational medicine, and streamline the process by which clinical applications are established.

In an industry that is becoming increasingly specialised and technologically complex, the ability to utilize the collective intelligence of industrial peers is paramount to translational efficiency. Since no individual can acquire and apply the knowledge that may be provided by a collective audience, as medical treatment becomes more personalised, clinical decisions must be more experience and evidence based than ever before. Establishing a medium of collaborative communication between individual care providers in the translational pathway is essential to this process. Furthermore, collective intelligence provides a unique opportunity for the validation of emerging novel medical technologies and matching medical product development to current market and population health needs (2).

Therefore collective intelligence and crowdsourcing applications are increasingly relevant in order to help initiate and facilitate the process of science translation.

\section{Barriers to science translation and present solutions}

Identifying the barriers to efficient translation has been a critical and indeed global research and development issue for the last decade, gaining much attention from the 
scientific community (3) (4) (5) (6). Table 1 outlines commonly cited causes for stagnation along the pathways of translational science. Here, we have categorized barriers into those associated with the practicalities of research, the hurdles generated by institutional organisations and limitations with regard to the research workforce.

The influence translational medicine could make on healthcare has also been recognized outside the biomedical community. The United Kingdom (UK) Government has identified the need to facilitate the progression of this field and has proposed a myriad of changes to address barriers presented in table 1 . In order to alleviate the financial burden of research, amendments to funding applications, tax credit schemes, value added tax and cost-sharing exemptions have been suggested. Furthermore, concerns over the limiting regulations imposed on researchers have been raised and suggestions made as to how to reduce these. Much focus has been directed at generating environments in which bioinformatics can be generated and applied in clinical-research settings by altering the infrastructure and policies of institutions involved in research or clinical practice (7). There have been similar strategies employed in the United States (US) (8).

Most notably, Government initiatives have led to the formation of collaborative partnerships between universities, hospitals and businesses in the form of multidisciplinary centres. These institutions foster an integrated and multi-faculty research and development environment; within which clinical directions are aligned and expertise shared in order to successfully bring new discoveries into clinical fruition. These include the Academic Health Science Centres (AHSCs) and NIHR (National Institute for Health Research) Biomedical Research Centres and Units (BRCs and 
BRUs). This is an internationally recognised model employed by the American National Institutes for Health (NIH) in the form of National Centers for Advancing Translational Sciences (NCATS).

In order to reap the benefits of interdisciplinary centres, it has been suggested that Master's or Certificates in Advanced Education in Translational Medicine should be introduced (9). The courses would provide experience of product development, technological management, market positioning and regulation, training advocates in translational medicine. Individuals with such skill sets would be able to identify metrics of both efficacy assessment and sustainability of translational medicine and incentivize direction towards the production of clinical applications (10).

Despite these efforts, issues such as career pathways, academic rewards, differing economic incentives, departmental budgets and policies still prevent streamlined science translation (11) (12) (13). With these hurdles persisting, a new infrastructure is required to take advantage of scientific and technological advances.

\section{Collective intelligence and crowdsourcing}

The ubiquitous use of the Internet proffers a new dimension of connection and collaboration within the biomedical community. The use of online networks to enable access to the cumulative knowledge of individual contributors is termed collective intelligence (CI). Common examples of this model include Wikipedia, YouTube and open-source software such as Linux. Actively directing this body of knowledge towards a specific creative or practical problem is a tactic known as crowdsourcing 
(14) (15). These two methods can be used in conjunction with one another. Whilst CI increases the capacity for knowledge, crowdsourcing broadens the diversity of input and offers exposure to a variety of specialist skills. The paradigms of 'crowd wisdom' and 'marginality' describe the increased insight and creativity brought to a developing team through the combination of distinguished cognitive heuristics from multiple fields. These attributes have made CI and crowdsourcing methods popular business tools (16). By implementing these methods within translational pathways, opportunities provided by modern science could be seized to drive unmatched advances in medical care.

CI has already been recognized as a successful method in facilitating innovation and has been applied within several different industries. Crowdsourcing instructs the outsourcing of large-scale data acquisition from dispersed locations and as a result has been employed within the public health domain. The US Geological Society (USUG) has developed an earthquake mapping application, 'Did You Feel it?' which relies on data input from citizens regarding their location and the extent to which they felt the tremor. Thus allowing for damage predictions and appropriate disaster relief dissemination (17).

The use of web interfaces within the healthcare sector is expanding (18). Personalised health care apps which support lifestyle changes such as smoking cessation (19), weight loss (20), exercise programs and the management of chronic pain disorders are gaining popularity(21). The MyHeartMap Challenge tasks citizens with the programming of a dynamic map of defibrillators within the community for emergency use, via a smart phone application (22). The success of these applications highlights 
the acceptance of modern technology within the healthcare domain and supports the use of online and technological software within this field.

Despite their use within the healthcare sector, CI and crowdsourcing have not yet been fully embraced as a method of improving public health through their application within translational medicine.

Applications of collective intelligence and crowdsourcing for healthcare innovation

Applying the CI of a multi-disciplinary audience and using an online interface to procure, present and process data on a large scale through crowd-sourcing applications offers a new framework to accelerate translational medicine. The lack of geographical, institutional and economic barriers could sanction healthcare development worldwide, breeching the long-standing issues that currently impede translational progression. These methods can support multi-disciplinary collaboration and be directed towards multiple applications from solving empirical research problems, to data acquisition and analysis, or education.

\section{$\underline{\text { Solving empirical research problems }}$}

Modern technology has provided the research community with data on a monumental scale, for example the Human Genome Project. However empirically provable problems often remain unsolved due to time, labor, financial, or political constraints, 
leaving research questions unanswered. Crowdsourcing can be used to overcome this issue by outsourcing tasks to a specific audience. A notable and successful example is Foldit; a project that allows users to help identify the unknown motif of folded proteins that can be used to find cures for diseases such as Human Immuno deficiency Virus (HIV) and potentially reduce mortality and morbidity worldwide (23).

Similarly, DREAM (Dialogue for Reverse Engineering Assessments and Methods) and the non-profit Prize4Life group pioneered a crowdsourcing competition in which contributors were challenged with the identification of algorithms to monitor the progression of Amytrophic Lateral Sclerosis (ALS), a disease with highly variable presentations and progression patterns. The result of this competition was the production of thirty-seven unique algorithms, two of which outperformed those suggested by ALS clinicians. In addition several potential new biomarkers of disease progression were highlighted, further elucidating the pathophysiology behind ALS and aiding the diagnosis of 'fast' versus 'slow' disease types (24). The FoldIt and ALS challenge projects demonstrate the potential for crowdsourcing data and benefitting from the collective intelligence of an audience, illustrating how these methods can be employed to answer empirical research questions and to enhance and accelerate science translation.

\section{Data acquisition, access and analysis}

Whilst traditional data acquisition can be time and labor intensive, crowdsourcing can be used to obtain patient data from diverse, heterogeneous populations. Despite the necessity of defined inclusion and exclusion criteria, the subject population involved 
in clinical trials is often recruited from a small geographical area within the locality of the research facility, which depending on the consistency of the population may lead to the acquisition of data from an unrepresentative sample population. The collation of data from multiple research centres can overcome this problem to an extent, although data acquisition from remote populations but may remain limited. Using an online interface to gather information accelerates data acquisition and provides any patient or subject with the opportunity to contribute on a global scale, regardless of where they live and what kind of healthcare they have access to. However in this case reliable internet access and local motivation to join a trial are limiting factors.

Several studies have compared data collected by traditional face-to-face consultations and online crowdsourcing methods (25) (26). These identified the huge potential of fast and diverse data retrieval crowdsourcing proffered. Improving the nuances of online data acquisition and designing validated outcome measurements to ensure accuracy and improve the validity of data provided could lead to the popularisation of online clinical trials which accumulate data from an international sample group (27). The use of this type of data retrieval lends it self most favorably to subjective data collection as this is unaffected by incorrect estimations or incomplete measurements of confounders by patient contributors. This would be valuable to both individual users and those tasked with informing clinical or health policies in the future and could lead to revolutionary change in the format and progression of clinical trials.

Departmentalized institutional silos often limit access to data that has already been collated by other research groups. To overcome this online, open-access platforms, presenting data submitted from multiple faculties within the biomedical community 
have been set-up, facilitating the analysis of previously obtained data and reducing the costs of research and providing researches access to data they might not be able to acquire otherwise.

Several online networks are developing as platforms for open-access data. In the UK the Clinical Practice Research Datalink (CPRD) collates primary care data and makes it available to researchers. Retrospective, observational cohort analysis studies are being conducted on the basis of this data, facilitating the manipulation of one large data set for several, unrelated research topics (28) (29). A similar network exists across the CTSA collaborations in the US (13).

The Prostate Cancer DREAM challenge was launched with the aim of improving predictive models of disease progression and treatment toxicity in prostate cancer by mining historical data (30). The data was originally compiled by the Project Data Sphere, a collaborative platform committed to providing open-access and analysis of historical phase III oncology data sets. The predictions identified through this project could reduce the redundancy of historical trial data, aid clinical decisions and improve patient outcomes in metastatic prostate cancer patients and exemplifies the role of open-access data sourced from a multi-disciplinary contributors in accelerating translational medicine.

HipSci, the UK's induced pluripotent stem cell (iPS) resource is a more specialized example that collates genomic, proteomic and cell biology data, thus enabling research into the effects of genomic variation of cellular phenotype and provides the opportunity to elucidate new disease mechanisms (31). This data bank has affiliations 
with the UK's National Health Service in order to provide corresponding clinical information and therefore can be used to develop understanding of genotype on disease phenotype and illuminating novel therapeutic approaches.

The pharmaceutical industry has also implemented crowdsourcing applications through the generation of public-private partnerships (PPPs), which are designed to aid the development of new molecular entities (NMEs) through the collaboration and sharing of data. One such partnership, Arch2POCM, uses a widely distributed network of biomedical professionals to identify the effects of certain drugs in a phase II clinical setting and seeks to establish proof of clinical mechanism (POCM) by establishing the molecular interactions of a drug and its effects on a biochemical level (32). Importantly, the data is rapidly published and freely available, regardless of whether the results are positive or negative and done so without pursuit of patents.

This 'precompetitive' method of drug discovery and development supports the performance of POCM tests within the public domain, reducing repetition of research within separate companies and thereby avoiding economic waste and redundancy of expertise time. In addition reduced restrictions can speed up the process and reveal the full benefits of certain drugs by validating new therapeutic targets and identifying pioneering applications of pre-existing medicines within an industrially sustainable model.

The Collaborative Chronic Care Network, C3Nproject, set up by researchers and physicians invites patients with inflammatory bowel disease to contribute data online, in order to improve the delivery of care and patient experience. The personalized 
learning system offers three domains: 1) social interaction between contributors, 2) technical support for data storage and analysis and 3) an environment to test new treatment ideas, and as a result facilitates new therapeutic ideas and improves treatment outcomes (33). This is an example of how crowdsourcing data acquisition and analysis can be paired with online forums and communication platforms to track chronic diseases on a personalised level and refine treatment using real-time data from patients, which is then accessible to researchers for future use.

\section{$\underline{\text { Education }}$}

As an increasingly global market, the learning environment can now extend beyond the classroom, university lecture theatre and indeed national boundaries. Crowdsourcing applications have been used to facilitate medical learning, overcoming barriers of location by providing all students with the opportunity to observe new techniques and distribute new policies in clinical skills. WebSurg is an online library of surgical videos depicting surgical techniques from all specialties. It functions as a 'virtual surgical university' and aims to encourage integrative learning between students, surgeons, anesthetists and nurses, mediating training, information distribution and peer review exchanges globally (34). Facilitating inter-professional education provides experience of multi-disciplinary relationships and provides students with skill sets amenable to collaborative work in the future, which may overcome the current limitation of lacking a collaborative translationalist work force.

Academia.edu is a forum upon which researchers can upload their research, track other researchers and their work and analyse the impact of their own work within 
their own field and others. This platform again exemplifies the role for collating interdisciplinary data and knowledge with open-access to accentuate learning and accelerate scientific translation (35).

\section{Perspectives in Collective Intelligence for Biomedical Science Translation}

The evidence supporting the use of CI and crowdsourcing is compelling, however many hurdles to their successful application remain. As the scientific and business communities continue to embrace these concepts, robust mechanisms to safeguard patient data anonymity will become increasingly important in order to allow dissemination and use within open-access platforms. Furthermore, diplomacy will be required in order to facilitate the integration between public and private sectors, which may have conflicting interests regarding the intellectual property of any commercially viable advances made through CI.

\section{Conclusions}

The recent implementation of crowdsourcing tools into the biomedical research community is just beginning to be appreciated; yet its potential is unique. The recent and exponential increase in the volume and complexity of clinical data tasks the research community to identify a new method of data analysis and necessitates networking. In order to facilitate much needed scientific advancement both collaboration, communication and comprehensive access to high quality data is required. By mediating interdisciplinary networking, platforms collating their 
collective intelligence can drive the development of new therapeutic options and improve the standard of medical care.

The examples discussed in this article represent the growing field of collective intelligence and crowdsourcing within biomedical innovation and demonstrate the diversity of their application. Using modern technology to mine the collective intelligence of research communities and create global networks to reinforce connections between stakeholders of the translational pathway can offer a solution to the current, reduced efficacy in the bench to bedside journey. Embracing these methods promotes a future for successful medical innovation and an opportunity to expedite the research-development-dissemination process and improve healthcare worldwide, as we enter a new era of medicine. 
1. Jalali M, Kirkpatrick WNA, Cameron MG, Pauklin S, Vallier L. Human stem cells for craniomaxillofacial reconstruction. Stem Cells Dev. 2014 Jul 1;23(13):143751.

2. Ijzerman MJ, Steuten LMG. Early assessment of medical technologies to inform product development and market access: a review of methods and applications. Appl Health Econ Health Policy. 2011 Sep 1;9(5):331-47.

3. Sussman S, Valente TW, Rohrbach LA, Skara S, Pentz MA. Translation in the Health Professions Converting Science into Action. Eval Health Prof. 2006 Mar 1;29(1):7-32.

4. Marincola FM. Translational Medicine: A two-way road. J Transl Med. 2003 Jul 24;1:1.

5. Pober JS, Neuhauser CS, Pober JM. Obstacles facing translational research in academic medical centers. FASEB J. 2001 Nov 1;15(13):2303-13.

6. Ioannidis JP. Materializing research promises: opportunities, priorities and conflicts in translational medicine. J Transl Med. 2004 Jan 31;2(1):5.

7. Department for Business, Innovation and Skills (BIS) (last), Department of Health. Strategy for UK Life Sciences oobj:. Office for Life Sciences; 2011.

8. Zerhouni E. The NIH Roadmap. Science. 2003 Oct 3;302(5642):63-72.

9. Kurpinski K, Johnson T, Kumar S, Desai T, Li S. Mastering Translational Medicine: Interdisciplinary Education for a New Generation. Sci Transl Med. 2014 Jan 8;6(218):218fs2-218fs2.

10. Staff NP, Runge BK, Windebank AJ. Breaking Down Translation Barriers: Investigator's Perspective. Sci Transl Med. 2014 Sep 3;6(252):252cm7-252cm7.

11. Rietschel ET, Bruckner-Tuderman L, Schütte G, Wess G. Moving medicine forward faster. Sci Trans1 Med. 2015 Mar 4;7(277):277ed2.

12. Edelman ER, LaMarco K. Clinician-Investigators as Translational Bioscientists: Shaping a Seamless Identity. Sci Transl Med. 2012 May 23;4(135):135fs14-135fs 14.

13. Heller C, de Melo-Martín I. Clinical and Translational Science Awards: Can They Increase the Efficiency and Speed of Clinical and Translational Research?: Acad Med. 2009 Apr;84(4):424-32.

14. Howe, J. The rise of crowdsourcing. [Internet]. 2006. Available from: www.wired. com/wired/archive/14.06/crowds.html

15. Brabham, DC. Crowdsourcing as a model for problem solving: an introduction and cases. Converg Int J Res New Media Technol. 2008;14(1):75-90.

16. Surowiecki J. The wisdom of crowds: why the many are smarter than the few and how collective wisdom shapes business, economies, societies, and nations. New York: Doubleday; 2004.

17. Atkinson, Gail M., Wald, David J. "Did You Feel It?” Intensity Data: A Surprisingly Good Measure of Earthquake Ground Motion. Seismol Res Lett. 2007 Jun;78(Number 3):362-8.

18. Brabham DC, Ribisl KM, Kirchner TR, Bernhardt JM. Crowdsourcing applications for public health. Am J Prev Med. 2014 Feb;46(2):179-87.

19. Strecher VJ, Shiffman S, West R. Randomized controlled trial of a web-based computer-tailored smoking cessation program as a supplement to nicotine patch therapy. Addict Abingdon Engl. 2005 May;100(5):682-8.

20. Burke LE, Styn MA, Sereika SM, Conroy MB, Ye L, Glanz K, et al. Using mHealth technology to enhance self-monitoring for weight loss: a randomized trial. Am J Prev Med. 2012 Jul;43(1):20-6. 
21. Rosser BA, Eccleston C. Smartphone applications for pain management. J

Telemed Telecare. 2011;17(6):308-12.

22. McGill N. Online-only: Crowdsourcing lets smartphone users identify where to jumpstart a heart. Nations Health. 2013 Jan 1;42(10):E53-E53.

23. Cooper S, Khatib F, Treuille A, Barbero J, Lee J, Beenen M, et al. Predicting protein structures with a multiplayer online game. Nature. 2010 Aug 5;466(7307):756-60.

24. Küffner R, Zach N, Norel R, Hawe J, Schoenfeld D, Wang L, et al. Crowdsourced analysis of clinical trial data to predict amyotrophic lateral sclerosis progression. Nat Biotechnol. 2015 Jan;33(1):51-7.

25. Armstrong AW, Cheeney S, Wu J, Harskamp CT, Schupp CW. Harnessing the power of crowds: crowdsourcing as a novel research method for evaluation of acne treatments. Am J Clin Dermatol. 2012 Dec 1;13(6):405-16.

26. Armstrong AW, Harskamp CT, Cheeney S, Wu J, Schupp CW. Power of crowdsourcing: novel methods of data collection in psoriasis and psoriatic arthritis. $\mathrm{J}$ Am Acad Dermatol. 2012 Dec;67(6):1273-81.e9.

27. Swan M. Crowdsourced health research studies: an important emerging complement to clinical trials in the public health research ecosystem. J Med Internet Res. 2012;14(2):e46.

28. Macedo AF, Douglas I, Smeeth L, Forbes H, Ebrahim S. Statins and the risk of type 2 diabetes mellitus: cohort study using the UK clinical practice pesearch datalink. BMC Cardiovasc Disord. 2014;14:85.

29. Pfeil AM, Imfeld P, Pettengell R, Jick SS, Szucs TD, Meier CR, et al. Trends in incidence and medical resource utilisation in patients with chronic lymphocytic leukaemia: insights from the UK Clinical Practice Research Datalink (CPRD). Ann Hematol. 2015 Mar;94(3):421-9.

30. Abdallah K, Hugh-Jones C, Norman T, Friend S, Stolovitzky G. The Prostate Cancer DREAM Challenge: A Community-Wide Effort to Use Open Clinical Trial Data for the Quantitative Prediction of Outcomes in Metastatic Prostate Cancer. The Oncologist. 2015 May;20(5):459-60.

31. Viswanathan P, Gaskell T, Moens N, Culley OJ, Hansen D, Gervasio MKR, et al. Human pluripotent stem cells on artificial microenvironments: a high content perspective. Front Pharmacol [Internet]. 2014 Jul 2 [cited 2015 May 29];5. Available from: http://www.ncbi.nlm.nih.gov/pmc/articles/PMC4078252/

32. Norman TC, Bountra C, Edwards AM, Yamamoto KR, Friend SH. Leveraging Crowdsourcing to Facilitate the Discovery of New Medicines. Sci Transl Med. 2011 Jun 22;3(88):88mr1-88mr1.

33. Margolis PA, Peterson LE, Seid M. Collaborative Chronic Care Networks (C3Ns) to Transform Chronic Illness Care. Pediatrics. 2013 Jun;131(Suppl 4):S21923.

34. Mutter D, Vix M, Dallemagne B, Perretta S, Leroy J, Marescaux J. WeBSurg: An innovative educational Web site in minimally invasive surgery--principles and results. Surg Innov. 2011 Mar;18(1):8-14.

35. Academia.edu [Internet]. Academia.eu. 2015 [cited 2015 Jul 8]. Available from: https://www.academia.edu/about 
Table I. Current barriers to efficient science translation (13)

\begin{tabular}{|c|c|}
\hline Type of barrier & Barriers to effective translation \\
\hline Practicalities of research & $\begin{array}{l}\text { Difficulty in recruiting volunteers for clinical trials } \\
\text { Poor outreach and access to minority groups as a volunteer base } \\
\text { Incompatible data formats between research and clinical databases } \\
\text { Expenses and lack of funding } \\
\text { Clinical applications in demand are often not the most economically viable }\end{array}$ \\
\hline Organisational hurdles & $\begin{array}{l}\text { No co-ordinated inter-disciplinary collaboration } \\
\text { Departmental budgeting creates barriers to integrative work } \\
\text { Policies and procedures differ across faculties, industries and borders } \\
\text { Institutional regulation limits progression }\end{array}$ \\
\hline Collaborative team & $\begin{array}{l}\text { Lack of qualified 'translationists', career pathways and poor career incentives in this area } \\
\text { Clinical goals of researcher, clinician and developer often do not align } \\
\text { No designated research time for research-clinicians }\end{array}$ \\
\hline
\end{tabular}


Budge et al., 2015 